\section{CARACTERIZAÇÃO DE INFECÇÕES PULMONARES NO PÓS-OPERATÓRIO TARDIO DE TRANSPLANTE RENAL: UMA REVISÃO INTEGRATIVA}

\author{
Characterization of pulmonary infections in the late \\ postoperative period of kidney transplantation: an integrative \\ review
Caracterización de infecciones pulmonares en el postoperatorio tardío de trasplante renal: una revisión integrativa

Artigo de Revisão

\section{RESUMO}

Objetivo: Caracterizar a natureza e frequência das infecções pulmonares em pacientes adultos pós-transplantados renais tardios. Métodos: Foi realizado um estudo de revisão bibliográfica nas seguintes bases de dados eletrônicas: PubMed, Scielo e Web of Science. Os critérios de elegibilidade do estudo foram: artigos publicados entre os anos de 2010 e 2015, nos idiomas inglês, português ou espanhol, do tipo: ensaios clínicos, randomizados ou não, estudos de caso-controle, estudos de coorte e estudos longitudinais em humanos. Foram excluídos os artigos cujos participantes da pesquisa fossem menores de 18 anos de idade, além dos artigos duplicados em mais de uma das bases de dados. As palavras-chave utilizadas e combinadas na pesquisa foram: pneumonia, infecção pulmonar, infecção, transplante renal, hospitalização. Resultados: Os agentes etiológicos mais incidentes são Pneumocystis jirovecii, Mycobacterium tuberculosis e Aspergillus fumigatus. As infecções pulmonares são devidas, em grande número, ao regime imunossupressor, tempo prolongado de hemodiálise, disfunção de enxerto e transmissão inter humana. Frequentemente, essas infecções evoluem com dispneia progressiva e insuficiência respiratória aguda, sendo necessária ventilação mecânica invasiva ou não invasiva. Conclusão: As evidências científicas apontam uma alta prevalência de infecções pulmonares nos pacientes transplantados renais.

Descritores: Hospitalização; Transplante de Rim; Infecções Respiratórias.

\section{ABSTRACT}

Objective: The aim of this study was to characterize the nature and frequency of pulmonary infections in late post-kidney transplant adult recipients. Methods: A bibliographic review was conducted in the following electronic databases: PubMed, SciELO and Web of Science. The study eligibility criteria were articles published between the years 2010 and 2015, in English, Portuguese or Spanish, comprising clinical trials, randomized or not, case-control studies, cohort studies, and longitudinal studies in humans. Articles whose research subjects were aged under 18 years were excluded, as well as repeated articles, which appeared in more than one of the databases. The keywords used and combined in the research were: pneumonia, lung infection, infection, kidney transplantation, hospitalization. Results: The most common etiological agents are Pneumocystis jirovecii, Mycobacterium tuberculosis and Aspergillus fumigatus. Pulmonary infections are, in a large number, due to the immunosuppressive regimen, extensive length of time on hemodialysis, graft dysfunction and interhuman transmission. Often, such infections evolve with progressive dyspnea and acute respiratory failure, thus requiring invasive or non-invasive mechanical ventilation. Conclusion: The evidences point out a high prevalence of pulmonary infections in kidney transplant recipients.

Descriptors: Hospitalization; Kidney Transplantation; Respiratory Tract Infections.
Camilla Tibiriçá da Silva Borges $^{(1)}$

Vinícius Augusto Travassos ${ }^{(1)}$ Luciana Castilho de Figueiredo ${ }^{(1)}$ Desanka Dragosavac ${ }^{(1)}$ Daniela Cristina dos Santos $\operatorname{Faez}^{(1)}$ Ana Isabela Morsch Passos ${ }^{(1)}$

1) Universidade Estadual de Campinas UNICAMP - Campinas (SP) - Brasil.

Recebido em: 24/02/2016 Revisado em: 01/03/2016 Aceito em: 16/03/2016 


\section{RESUMEN}

Objetivo: El objetivo del estudio fue caracterizar la naturaleza y la frecuencia de las infecciones pulmonares de pacientes adultos pos trasplante renal tardio. Métodos: Se realizó un estudio de revisión bibliográfica en las siguientes bases de datos electrónicas: PubMed, Scielo y Web of Science. Los criterios de elegibilidad del estudio fueron: artículos publicados entre los años de 2010 y 2015 en los idiomas inglés, portugués o español, del tipo ensayos clínicos randomizados o no, estudios de casocontrol, estudios de cohorte y estudios longitudinales en humanos. Fueron excluidos los artículos cuyos los participantes de la investigación tenían menos de 18 años de edad, además de los artículos repetidos en más de una base de datos. Las palabrasclave utilizadas y asociadas en la investigación fueron: neumonía, infección pulmonar, infección, trasplante renal, hospitalización. Resultados: Los agentes etiológicos más incidentes son la Pneumocystis jirovecii, el Mycobacterium tuberculosis y el Aspergillus fumigatus. Las infecciones pulmonares son causadas, en su mayoría, por el régimen inmunosupresor, el largo tiempo de hemodiálisis, la disfunción del injerto y la trasmisión inter humanos. Esas infecciones evolucionan, con frecuencia, con disnea progresiva e insuficiencia respiratoria aguda y la necesidad de ventilación mecánica invasiva o no invasiva. Conclusión: Las evidencias cientificas apuntan elevada prevalencia de infecciones pulmonares en los pacientes trasplantados renales.

Descriptores: Hospitalización; Trasplante de Riñon; Infecciones del Sistema Respiratorio.

\section{INTRODUÇÃO}

O transplante de órgãos sólidos mais comum no mundo é o transplante renal, sendo esSe o tratamento de escolha para a doença renal terminal. No Brasil, é o tipo de transplante mais realizado - entre janeiro e junho de 2015, foram feitos 2664 transplantes renais ${ }^{(1)}$. Comparado à diálise crônica, o transplante renal é rentável, oferece melhora na qualidade de vida e confere um benefício progressivo à sobrevida do paciente. A taxa de sobrevida dos enxertos aumentou consistentemente durante as últimas décadas ${ }^{(2)}$.

O emprego de imunossupressão em pacientes póstransplantados renais pode predispor ao aparecimento de infecções oportunistas, tais como as respiratórias ${ }^{(3)}$. Devido à imunossupressão, os pacientes com enxertos funcionantes são expostos a variados patógenos, gerando impacto na morbidade e mortalidade, explicando por que a infecção é a segunda causa de morte em pacientes transplantados renais ${ }^{(1)}$. As infecções pulmonares, como pneumonia e infecções do trato respiratório inferior, estão entre as complicações pós operatórias, juntamente com as complicações não infecciosas, tais como atelectasias, derrame pleural e disfunção diafragmática.
Durante o período de máxima imunossupressão (dentro de seis meses após o transplante), o risco de ocorrência de vírus e agentes oportunistas é alto. De todas as infecções, $20 \%$ são pulmonares. As drogas imunossupressoras podem induzir um efeito negativo nos pulmões, como a toxicidade, principalmente em transplantados renais, levando à pneumonite intersticial, bronquiolite obliterativa e pneumonia ${ }^{(4)}$.

Este estudo teve como objetivo caracterizar a frequência e a natureza das infecções pulmonares em pacientes adultos pós-transplantados renais tardios.

\section{MÉTODOS}

Trata-se de uma revisão bibliográfica, na qual foi realizada uma busca nas seguintes bases de dados eletrônicas: PubMed, Scielo e Web of Science.

Os critérios de elegibilidade do estudo foram: artigos publicados entre os anos de 2010 e 2015, nos idiomas inglês, português ou espanhol, do tipo: ensaios clínicos, randomizados ou não, estudos de caso-controle, estudos de coorte e estudos longitudinais, realizados em humanos. Foram excluídos os artigos cujos sujeitos da pesquisa fossem menores de 18 anos de idade, além duplicados em mais de uma das bases de dados.

As palavras-chave utilizadas e combinadas na pesquisa foram: pneumonia, infecção pulmonar, infecção, transplante renal, hospitalização e seus correspondentes nos idiomas inglês e espanhol. Em seguida, procedeu-se à análise dos artigos selecionados, realizada por dois pesquisadores por meio da leitura do título, resumo e texto completo, respectivamente.

Os desfechos analisados foram: tipo de estudo, ano de publicação, número de pacientes, local de estudo, instrumento utilizado para coleta de dados, tempo de transplante, o agente etiológico da infecção, tempo de internação, tempo e/ou necessidade de ventilação mecânica, taxa de óbitos, detalhes da evolução dos quadros, se houve evolução para quadro de Síndrome do Desconforto Respiratório Agudo (SDRA) e se foi realizada manobra de recrutamento alveolar.

\section{RESULTADOS E DISCUSSÃO}

Após análise conjunta dos pesquisadores e identificação dos critérios de inclusão e exclusão expostos, foram selecionados 77 artigos. Destes, 22 artigos foram excluídos (3 por não serem artigos científicos e 19 por falta de relação com o tema a ser discutido), restando para leitura e análise um total de 55 artigos, conforme fluxograma a seguir (Figura 1). As características demográficas dos pacientes em seus 
respectivos estudos estão listadas na Tabela I, na qual apenas não foram incluídos três artigos selecionados, classificados como revisão de literatura. O restante dos resultados será apresentado em tópicos, para melhor elucidação do leitor.

Figura 1 - Fluxograma de seleção dos artigos científicos.

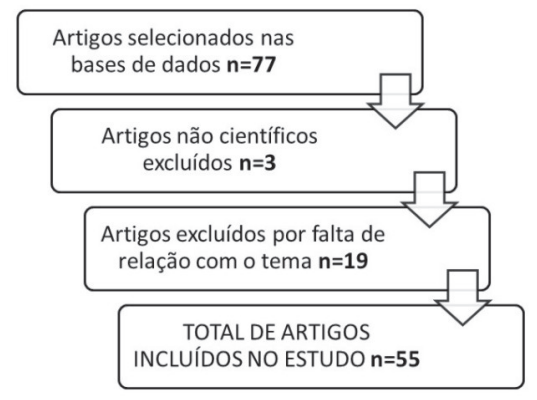

Os tipos de estudo encontrados foram relatos de caso $^{(9,1}$ $2,17,22,25,30,37,41,42,44,47,50,51,57,58)$, estudos retrospectivos ${ }^{(5,7,8,10,11,21,24}$ ,28,29,31,32,35,39,43,45,56) , coorte ${ }^{(13,14,18,19,23,27,33,34,36,38,40,46,48,49,55)}$, casocontrole $^{(6,15,20,26,59)}$ e revisão de literatura ${ }^{(16,33,52)}$. Os locais de pesquisa dos artigos selecionados foram China ${ }^{(9,11,45,46,50)}$, Espanha $^{(12,14,38,39,58)}$, Tunísia ${ }^{(33)}$, Bélgica ${ }^{(57)}$, Dinamarca ${ }^{(15,19)}$,
Malásia $^{(17)}$, Alemanha ${ }^{(7,18,23,28)}$, Turquia ${ }^{(21,25,32,34,36)}$ Brasil $^{(22)}$, Eslovênia $^{(24)}$, Holanda ${ }^{(26,27,55)}$, Taiwan ${ }^{(8,31,49,56)}$ França $^{(5,6,29,43)}$,

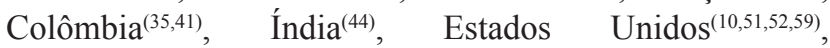
Portugal $^{(30)}$, Itália ${ }^{(42)}$, Austrália ${ }^{(47)}$, México $^{(48)}$ e Grécia $^{(40)}$. Nota-se que, pelos critérios de inclusão estabelecidos, foi encontrado apenas um estudo desenvolvido por pesquisadores brasileiros, ressaltando-se a necessidade do progresso científico nacional nessa temática.

\section{Agentes etiológicos}

Os agentes etiológicos mais comuns encontrados nos artigos selecionados foram Pneumocystis jirovecii ${ }^{(5-30)}$, Mycobacterium tuberculosis ${ }^{(29,31-36)}$ e Aspergillus fumigatus $^{(10,14,21,32,37-41)}$ A seguir, serão expostos, em separado, resultados sobre estes três principais agentes etiológicos.

As técnicas relatadas para o diagnóstico dos agentes etiológicos foram: broncoscopia com lavado broncoalveolar ${ }^{(5,6,9,10,12-14,17,19-22,25,26,29,30,32,36,38,39,41-54)}$, reação em cadeia da polimerase $e^{(6-8,14,15,17-20,22,23,25,27-29}$, 43-45,49,52), exame histopatológico ${ }^{(28,35,37,55)}$, biópsia pulmonar $^{(10,33,36,42,45,51,53,56)}$, radiografia e tomografia computadorizada ${ }^{(7,9-12,21,22,29,34,41-43,45,47,49,51,54-58) \text {, técnica }}$ de Ziehl-Neelsen ${ }^{(21,25,33,41,53,57)}$, técnica de $\operatorname{Gram}^{(41)}$ e imunofluorescência ${ }^{(15,17,27)}$.

Tabela I - Características gerais de 52 dos 55 estudos selecionados. Campinas, SP, 2010-2015.

\begin{tabular}{|c|c|c|c|c|c|}
\hline Autor/ano & $\mathbf{n}$ & $\begin{array}{l}\text { Idade em anos } \\
\text { (média } \pm \text { DP) }\end{array}$ & $\begin{array}{c}\text { Tempo de } \\
\text { Internação de } \\
\text { UTI em dias }\end{array}$ & $\begin{array}{c}\text { Necessidade } \\
\text { de VM } \\
\text { (quantitativo) }\end{array}$ & Óbitos (\%) \\
\hline Brunot V. et al., 2012(5) & 9 & NI & 8 a 15 & 3 & NI \\
\hline Le Gal S. et al., $2012^{(6)}$ & 18 & 62 & NI & NI & NI \\
\hline Pliquett RU. et al., 2012(7) & 29 & $54,9 \pm 14,9$ & NI & NI & 34 \\
\hline Yang CY. et al., 2012(8) & 20 & $54,5 \pm 13,0$ & NI & 14 (IRpA) & 50 (IRpA) \\
\hline Tu GW. et al., 2013(9) & 3 & 46,3 & 35 & NI & 33 (SEPSE) \\
\hline Chen G. et al., 2010 & $\begin{array}{l}53 \text { casos } \\
\text { e } 2520 \\
\text { controles }\end{array}$ & $\begin{array}{c}47,3 \pm 13,1 \text { anos - } \\
\text { casos } \\
42,6 \pm 12,5 \text { anos - } \\
\text { controles }\end{array}$ & NI & NI & 45 (PNM) \\
\hline Song T. et al., 2010 $0^{(11)}$ & 13 & 37 & NI & NI & NI \\
\hline Perez-Ordoño L. et al., 2014(12) & 4 & 50,5 & 21 & 4 & NI \\
\hline Phipps LM. et al., 2011 $1^{(13)}$ & 14 & $46,6 \pm 13.2$ & NI & $10(\operatorname{IRpA})$ & 14 (PNM) \\
\hline Hoyo I. et al., 2012(14) & 1656 & 51 & $\mathrm{NI}$ & NI & $1.5(\mathrm{PNM})$ \\
\hline Rostved AA. et al., 2013 ${ }^{(15)}$ & $\begin{array}{c}16 \text { casos e } 32 \\
\text { controles }\end{array}$ & $\begin{array}{c}46 \text { anos - casos } \\
46 \text { anos - controles }\end{array}$ & 25 & NI & NI \\
\hline Boer MGJ. et al., 2011 (16) & $\begin{array}{c}50 \text { casos e } 99 \\
\text { controles }\end{array}$ & $\begin{array}{l}57,4 \text { anos - casos } \\
52,1 \text { controles }\end{array}$ & NI & 4 & $6(\mathrm{PNM})$ \\
\hline Iqbal MAH. et al., 2012 $2^{(17)}$ & 1 & 38 & NI & NI & NI \\
\hline Fritzsche C. et al., 2013 ${ }^{(18)}$ & 70 & $55,0 \pm 12$ & NI & $\mathrm{NI}$ & NI \\
\hline Leth S. et al., 2014 ${ }^{(19)}$ & 46 & 59,2 & NI & NI & NI \\
\hline
\end{tabular}




\begin{tabular}{|c|c|c|c|c|c|}
\hline De Castro N. et al., 2010 & 11 & 53 & NI & NI & 0,9 (PNM) \\
\hline Dizdar OS. et al., 2014(21) & 82 & $38 \pm 12$ & NI & NI & 34 (PNM) \\
\hline Ramalho J. et al., 2014(22) & 1 & 54 & NI & NI & NI \\
\hline Maruschke M. et al., 2014 & 7 & $64,3 \pm 13,9$ & NI & NI & 42 (PNM) \\
\hline Borstnar S. et al., 2013 ${ }^{(24)}$ & 13 & $49 \pm 4$ & NI & NI & 23 (PNM) \\
\hline Metan G. et al., 2011 ${ }^{(25)}$ & 1 & 40 & 25 & NI & NI \\
\hline Struijk GH. et al., 2011 & 9 & 56 & NI & NI & NI \\
\hline Eitner F. et al., 2011 ${ }^{(28)}$ & $\begin{array}{l}60 \text { casos e } 60 \\
\text { controles }\end{array}$ & $\begin{array}{c}53,9 \pm 13,9-\text { casos } \\
51,7 \pm 13,5- \\
\text { controles }\end{array}$ & NI & 23 & 26 (PNM) \\
\hline Bige N. et al., 2014 $4^{(29)}$ & 83 & 55.6 & 19 & 32 & 37 (PNM) \\
\hline Bento C. et al., 2014(30) & 1 & 51 & 22 & NI & NI \\
\hline Chen CH. et al., 2014 ${ }^{(31)}$ & 153 & $46,6 \pm 12,7$ & NI & NI & NI \\
\hline Eyüboğlu FÖ. et al., 2013 & 90 & $42,3 \pm 12,3$ & 19 & 27 (PNM) & 62 (PNM) \\
\hline Boubaker K. et al., 2013 ${ }^{(33)}$ & 16 & $32,2 \pm 12,7$ & NI & NI & $\begin{array}{l}6.2(\mathrm{~TB}) / 6.2 \\
\text { (SEPSE) }\end{array}$ \\
\hline Kupeli E. et al., 2011 ${ }^{(34)}$ & 136 & $36,3 \pm 12,2$ & NI & NI & NI \\
\hline Higuita LMS. et al., 2014 & 12 & 52,5 & 14 a 72 & NI & $16(\mathrm{~TB})$ \\
\hline Ersan S. et al., 2011 ${ }^{(36)}$ & 9 & $46,7 \pm 11,7$ & NI & NI & $11(\mathrm{~TB})$ \\
\hline Nasim A. et al., 2011 $1^{(37)}$ & 1 & 35 & NI & NI & NI \\
\hline Hoyo I. et al., $2010^{(38)}$ & 610 & 52,3 & NI & 15 & 1,4 (PNM) \\
\hline Hoyo I. et al., 2014(39) & 9 & 58,9 & NI & NI & NI \\
\hline Moloudi E. et al., 2012(40) & 27 & $42,7 \pm 12,3$ & 19 & 27 (PNM) & $62(\mathrm{PNM})$ \\
\hline Encarnación AA. et al., 2012 $2^{(41)}$ & 1 & 41 & 20 & NI & $100(\mathrm{PNM})$ \\
\hline Rizza V. et al., $2011^{(42)}$ & 1 & 32 & 10 & NI & NI \\
\hline Canet E. et al., 2011 $1^{(43)}$ & 200 & 56 & 10 & NI & NI \\
\hline Kute VB. et al., 2013 $3^{(44)}$ & 1 & 46 & NI & NI & NI \\
\hline Jiang T. et al., 2012(45) & 89 & 43.7 & NI & NI & NI \\
\hline Tu G. et al., 2015(46) & 60 & 53.5 & 20 & $21(\mathrm{IRpA})$ & $13(\operatorname{IRpA})$ \\
\hline Jabbar Z. et al., $2013^{(47)}$ & 1 & 70 & NI & NI & NI \\
\hline Valdez-Ortiz R. et al., 2011 & 350 & $37,96 \pm 11,4$ & NI & NI & 3.7 (PNM) \\
\hline Shih CJ. et al., 2014(49) & 33 & $56,9 \pm 11,4$ & 12 & 33 (PNM) & 45 (PNM) \\
\hline He Q. et al., 2011 & 2 & 48 & NI & NI & NI \\
\hline Cunha BA. et al., 2012(51) & 1 & 77 & 37 & NI & $100(\mathrm{PNM})$ \\
\hline Gainer SM. et al., 2012(52) & 18 & 46 & 18 & $4(\operatorname{IRpA})$ & $16(\mathrm{FO})$ \\
\hline Baas MC. et al., 2014 & 13 & 50 & NI & NI & NI \\
\hline Ou SM. et al., 2012(56) & 109 & $47,6 \pm 11,2$ & NI & NI & 22 (PNM) \\
\hline Ho TA. et al., $2010^{(57)}$ & 1 & 54 & 14 & NI & NI \\
\hline Fraile P. et al., $2013^{(58)}$ & 1 & 27 & NI & NI & NI \\
\hline Simkins J. et al., 2014 ${ }^{(59)}$ & $\begin{array}{l}13 \text { casos, } 39 \\
\text { controles }\end{array}$ & $\begin{array}{c}53 \pm 18 \text { casos, } 55 \pm \\
16 \text { controles }\end{array}$ & NI & NI & 46 (PNM) \\
\hline
\end{tabular}

DP: Desvio Padrão; FO: Falência de Órgãos; IRpA: Insuficiência Respiratória Aguda; n: Número de Pacientes; PNM: Pneumonia; TB: Tuberculose; UTI: Unidade de Terapia Intensiva; VM: Ventilação Mecânica Invasiva; NI: não informado pelo artigo. 


\section{Pneumocystis jirovecii}

A pneumonia causada pelo fungo Pneumocystis jirovecii $(\mathrm{PJ})$ é reconhecida como uma causa importante de morbimortalidade entre pacientes em uso de imunossupressores após transplante de órgãos sólidos. Transplantados renais possuem alto risco de infecção no período pós transplante, sobretudo nos dois primeiros anos, e uma taxa de mortalidade acima de $50 \%{ }^{(16,18,22-24,28)}$.

As causas de surtos de pneumonia por PJ e seus fatores de risco específicos ainda não estão claramente definidos. Há hipóteses de que um maior contato e transmissão inter-humana, reativação da infecção latente, exposição ambiental e análises mais sensíveis contribuam para a maior incidência de $\mathrm{PJ}^{(13,16,24)}$. Outros estudos sugerem como causas de $\mathrm{PJ}$ a transmissão por gotículas aéreas, baixa contagem de células-T CD4 e rejeição do enxerto ${ }^{(5,6,19,20)}$. Relata-se que a redução da imunidade devido ao tratamento imunossupressor e infecções por citomegalovírus também têm impacto no maior número de causas de pneumocistose em transplantados renais ${ }^{(15,27,30)}$. Outros fatores para o desenvolvimento de pneumonia por PJ incluem função renal deficiente e episódios de rejeição do enxerto ${ }^{(8,28)}$.

Os achados radiográficos e tomográficos mais evidentes após o diagnóstico de pneumonia por PJ foram infiltrados bilaterais difusos, padrão de vidro fosco e/ ou consolidação por todo o campo pulmonar ${ }^{(11,17,26)}$. O quadro clínico mais relatado é dispneia progressiva, hipoxemia, febre, tosse improdutiva e insuficiência respiratória aguda (IRpA), com frequente necessidade de intubação endotraqueal ${ }^{(12,20,25,26)}$. As causas mais comuns de IRpA em pacientes transplantados renais são pneumonia bacteriana, edema pulmonar cardiogênico e Síndrome do Desconforto Respiratório Agudo (SDRA). Esse quadro de comprometimento respiratório está associado à maior mortalidade e perda de enxerto, sendo imprescindível a profilaxia $^{(43)}$.

\section{Mycobacterium tuberculosis}

A tuberculose pulmonar por Mycobacterium tuberculosis (MT) é uma causa importante de mortalidade em países endêmicos, sendo sua incidência de 20 a 70 vezes maior em pacientes transplantados renais, principalmente devido ao seu estado imunossuprimido ${ }^{(33,35,53,60)}$. A transmissão de tuberculose através do doador já foi relatada em transplantes renais, hepáticos e pulmonares. A bactéria também pode ser adquirida após o transplante, com a taxa de infecção sendo maior em países em desenvolvimento ${ }^{(53)}$.

A tuberculose extrapulmonar ou disseminada tem igualmente maior ocorrência na população transplantada, se comparada à população em geral. Seus sintomas e manifestações clínicas são por vezes inespecíficos, o que torna difícil o diagnóstico precoce ${ }^{(36,61)}$. Os fatores de risco incluem o contato direto com portadores da bactéria, grupo sanguíneo $\mathrm{AB}$, hepatite $\mathrm{C}$, disfunção do enxerto, creatininemia, uso de imunossupressores, hemodiálise prolongada pré transplante, diabetes mellitus, doença pulmonar obstrutiva crônica (DPOC), doenças autoimunes e cirrose hepática ${ }^{(33,56)}$.

O quadro clínico caracteriza-se comumente por febre, perda de peso, síndrome pleurítica e infecção pulmonar resistente à antibioticoterapia. Os exames radiográficos mostram padrões miliares, derrame pleural, cavitações, nódulos pulmonares, consolidações, broncogramas aéreos e infiltrados pulmonares ${ }^{(33,35,36,53)}$. O diagnóstico da tuberculose, na prática, é tardio, sendo necessários, frequentemente, procedimentos invasivos como broncoscopia com lavado broncoalveolar ou biópsia do tecido pulmonar ou tecido envolvido $^{(53)}$.

\section{Aspergillus fumigatus}

A aspergilose é uma das infecções fúngicas oportunistas mais importantes em transplantados de órgãos sólidos, com alta taxa de mortalidade. A maioria dos casos ocorre dentro de 90 dias pós-transplante ${ }^{(14)}$. A incidência de infecções fúngicas, tais como a aspergilose, varia entre 1 e 14\%, com ocorrência de $74 \%$ no primeiro ano de transplante renal. $\mathrm{O}$ fungo Aspergillus fumigatus desenvolve quatro síndromes conhecidas: a aspergilose alérgica broncopulmonar, aspergiloma, aspergilose crônica necrotizante e aspergilose pulmonar invasiva. A aspergilose pulmonar pode progredir para uma doença invasiva, com mortalidade de cerca de $63 \%$. Os principais fatores de risco para a aspergilose são disfunção do enxerto, regime imunossupressor, insuficiência renal e necessidade de hemodiálise ${ }^{(37,41)}$.

O quadro clínico demonstra tosse produtiva, hemoptise, dor torácica, dispneia e febre. Os achados clínicos variam entre colonização de uma cavidade pulmonar prévia até invasão parenquimatosa e vascular. Radiologicamente, há envolvimento dos lobos superiores, cavitação, espessamento pleural, consolidação peribrônquica, áreas de padrão vidrofosco e nódulos centrolobulares ${ }^{(37,39)}$.

\section{Outros agentes etiológicos}

Outros agentes etiológicos citados em menor número e sem descrição dos fatores de risco para seu aparecimento, porcentagem de incidência, quadro clínico ou achados radiológicos são: Pseudomonas aeruginosa ${ }^{(21,29,32,38,43,46,49)}$, Staphylococcus $\quad$ aureus $^{(32,43,45,46,48,49)}, \quad$ Klebsiella pneumoniae $^{(32,46,49,59)}$, Acinetobacter baumanii ${ }^{(34,49)}$, Candida albicans ${ }^{(32,45,48)}$, Citomegalovirus ${ }^{(7,29,40,45)}$, Herpes simplex $^{(42,43,51)}$,Adenovirus ${ }^{(14)}$, Burkholderiapseudomallei $^{(47)}$, Escherichia coli $i^{(32,43,46,48)}, \quad \mathrm{H} 1 \mathrm{N1} 1^{(52,58)}, \quad$ Haemophilus 
influenzae ${ }^{(21)}$, Legionella pneumophila ${ }^{(38)}$, Lophomonas blattarum $^{(50)}$, M. catharralis $^{(32)}$, Mycobacterium avium $^{(57)}$, Nocardia spp ${ }^{(14)}$, Stenotrophomonas maltophilia ${ }^{(21,32)}$, Streptococcus hemolyticus ${ }^{(46)}$, Streptococcus pneumoniae $^{(32,34,38,43)}$,Enterococcus spp ${ }^{(48)}$, Mycobacterium intracellulare $^{(57)}$ e Varicella zoster ${ }^{(14)}$. Um estudo casocoorte avaliou a incidência e fatores de risco para a pneumonite induzida por everolimo em pacientes póstransplantados renais ${ }^{(55)}$. Dois artigos trouxeram a incidência de bronquiolite obliterante com pneumonia em organização associada a quadros infecciosos pulmonares ${ }^{(44,51)}$.

\section{Síndrome do Desconforto Respiratório Agudo}

A SDRA é uma patologia caracterizada por processo inflamatório, resultando em aumento de permeabilidade alvéolo capilar, com consequente edema intersticial e alveolar. Tem como principais sinais a hipoxemia severa, baixa complacência pulmonar e infiltrados bilaterais à radiografia de tórax ${ }^{(62)}$. A falência respiratória induzida pela SDRA tem alta taxa de mortalidade (de 50 a $70 \%$ ), sendo a maioria dos casos causada por sepse e terapia imunossupressiva $^{(58)}$. As infecções pulmonares predispõem ao surgimento de SDRA, sendo que em estudo realizado em 2015, dos 60 pacientes transplantados renais que desenvolveram pneumonia, 35 deles evoluíram com quadro de SDRA ${ }^{(46)}$.

Em estudo multicêntrico retrospectivo que incluiu 200 pacientes transplantados renais admitidos na Unidade de Terapia Intensiva (UTI) com IRpA, 15,5\% foram diagnosticados com SDRA. Os eventos de rejeição do enxerto e terapia imunossupressora, além das infecções pulmonares prévias, predispõe a um maior risco de desenvolvimento de SDRA $^{(43)}$. Segundo o estudo retrospectivo publicado em 2010, de 53 pacientes com micose pulmonar, 24 foram a óbito, em sua maioria devido à SDRA $(17 \%)^{(10)}$.

\section{Tempo de internação}

Não foram encontrados estudos que mostrem um tempo médio de internação para pacientes transplantados renais, devido a complicações e riscos que cada paciente pode desenvolver com o tempo. Muitos fatores podem colaborar com o risco de infecções, tais como o perfil de imunossupressão empregado, o cuidado pós-operatório e as exposições a diversas doenças infectocontagiosas. Além desses, o perfil socioeconômico desfavorável contribui para elevar a incidência destas complicações, em países em desenvolvimento ${ }^{(63)}$.

Episódios de infecções geralmente são mais comuns nos primeiros meses de acompanhamento do transplantado renal e estão diretamente ligados ao uso do imunossupressor utilizado, sendo os focos principais o trato urinário e ferida cirúrgica. Entre o segundo e sexto meses, as infecções oportunistas causadas por agentes virais e fúngicos predominam. Após o sexto mês, as infecções de origem comunitária predominam ${ }^{(64)}$.

\section{Ventilação mecânica e ventilação mecânica não invasiva}

As principais complicações pulmonares encontradas em pós-operatório são atelectasia, infecções traqueobrônquicas, pneumonia e insuficiência respiratória ${ }^{(65)}$. A resultante dessas complicações é um shunt arteriovenoso intrapulmonar, espessamento da membrana alveolar e inflamação do parênquima, com consequente edema e fibrose. Os pulmões tornam-se incapazes de fornecer oxigênio suficiente ao sangue, provocando dispneia grave, necessitando de ventilação mecânica invasiva ou ventilação mecânica não invasiva. Este quadro não revertido pode levar ao óbito ${ }^{(6))}$.

Foram ainda encontrados como relatos de complicações pulmonares em pacientes em pós operatório tardio de transplante renal a pneumonia adquirida na comunidade e Pneumonia Associada a Ventilação Mecânica (PAV), relatando a necessidade da utilização de ventilação mecânica ou ventilação mecânica não invasiva neste perfil de pacientes ${ }^{(40,49)}$. Alguns autores relatam em seus estudos que os pacientes receberam o tratamento de ventilação mecânica e ventilação mecânica não invasiva devido IRpA. A IRpA é uma disfunção respiratória causada por alterações na oxigenação ou na alimentação de $\mathrm{CO}_{2}$, que não satisfaz as demandas metabólicas do organismo em termos de trocas gasosas. A IRpA ocorrer por causas pulmonares ou por causas não pulmonares: SDRA, pneumonia, atelectasias, edema pulmonar, embolia pulmonar e DPOC ${ }^{(8,13,46,52)}$.

Alguns dos autores estudados citam ainda que os pacientes incluídos em seus respectivos estudos necessitaram de ventilação mecânica e ventilação mecânica não invasiva, não especificando os motivos para tal procedimento e não discutindo sobre o assunto ${ }^{(5,12,16,28,29,38)}$.

\section{Causas de óbito}

Dos 52 artigos selecionados, 27 descrevem as causas relacionadas ao óbito. A casuística sobre óbitos nos mostra um índice de mortalidade de $1 \%$ a $33 \%$, sendo que as causas principais foram pneumonia, sepse grave e tuberculose $\mathrm{e}^{(7-10,13,14,16,20,21,23,24,28,29,33,35,36,38-41,46,48,49,51,52,56,59)}$.

Há uma incidência de pneumonia por $P$. jirovecii em receptores de transplante de rim de $4 \%$, de coração de $4 \%$, de fígado de $11 \%$ e de coração e pulmão de $33 \%{ }^{(67)}$. O Pneumocystis jirovecii causa uma pneumonite intraalveolar, decorrente da ocupação dos espaços aéreos por exsudato rico em proteínas, podendo levar a complicações pulmonares e óbito ${ }^{(66)}$. 
A alta mortalidade por sepse grave e choque séptico está intimamente relacionada à abordagem do agente infeccioso. A conduta terapêutica, incluindo a antimicrobiana, vai diferir, substancialmente, de acordo com o local da infecção primária. A escolha inicial inadequada do esquema antimicrobiano pode levar a aumento significativo da taxa de mortalidade em pacientes sépticos ${ }^{(68)}$.

Apesar da potencial toxicidade do tratamento antituberculose em pacientes admitidos em Unidade de Terapia Intensiva (UTI), sugere-se que as medicações sejam iniciadas antes do resultado dos exames diagnósticos, visto que o atraso no início do tratamento pode resultar em óbito. Em pacientes imunossuprimidos, o índice de suspeita deve ser ainda maior. Os pacientes com tuberculose internados em UTIs podem desenvolver outras complicações, tais como pneumonia associada à VM, falência de múltiplos órgãos, choque séptico, insuficiência renal aguda, coagulação intravascular disseminada e sangramento digestivo. A insuficiência respiratória aguda causada por tuberculose e com necessidade de VM tem sido associada com taxas de mortalidade, entre $17,5 \%$ e $81,0 \%{ }^{(69)}$.

$\mathrm{O}$ presente estudo possui algumas limitações. Os estudos incluídos nesta revisão não relatam o motivo dos desfechos clínicos do paciente, sob os pontos de vista da necessidade de ventilação mecânica, do tempo de internação e porcentagem de óbitos, tornando difuso o conhecimento sobre estes aspectos neste perfil de pacientes. Sugerem-se estudos posteriores que complementem informações acerca da aplicação da ventilação mecânica e sobre o tempo de internação hospitalar ou em UTI, bem como as incidências de óbito.

\section{CONCLUSÃO}

Com base nos resultados obtidos, conclui-se que os agentes etiológicos mais incidentes em pacientes transplantados renais em regime imunossupressor são $P$. jirovecii, A. fumigatus e M. tuberculosis. A ocorrência de infecções pulmonares em transplantados renais é em grande parte devido ao uso de medicação imunossupressora, necessidade de hemodiálise prolongada, histórico de disfunção e/ou rejeição do enxerto e transmissão inter humana.

A frequência de infecção por estes agentes é alta, notadamente em pacientes imunossuprimidos, como o são os transplantados renais. Os sintomas mais comuns são dispneia progressiva e IRpA, sendo necessário ventilação mecânica invasiva e não invasiva. A evolução das infecções pulmonares pode gerar complicações severas como a SDRA, com elevada prevalência de óbito.

\section{REFERÊNCIAS}

1. Associação Brasileira de Transplante de Órgãos ABTO. Registro Brasileiro de Transplantes - RBT [Internet]. São Paulo; 2015 [acesso em 2015 Out 20]. Disponível em: http://www.abto.org.br/abtov03/ Upload/file/RBT/2015/rbt2015-1sem-lib2907.pdf

2. Carvalho MA, Freitas FGR, Silva HT Junior, Bafi AT, Machado FR, Pestana JOM. Mortality predictors in renal transplant recipients with severe sepsis and septic shock. PloS One. 2014;9(11):1-10.

3. Ewert R, Opitz C, Wensel R, Dandel M, Mutze S, Reinke P. Abnormalities of pulmonary diffusion capacity in long-term survivors after kidney transplantation. Chest. 2002;122(2):639-44.

4. De Gasperi A, Feltracco P, Ceravola E, Mazza E. Pulmonary complications in patients receiving a solidorgan transplant. Curr Opin Crit Care. 2014; 20(40: 411-9.

5. Brunot V, Pernin V, Chartier C, Garrigue V, Vetromile F, Szwarc I, et al. An epidemic of pneumocystis jiroveci pneumonia in a renal transplantation center: role of t-cell lymphopenia. Transplant Proc. 2012;44(9):281820.

6 .Le Gal S, Damiani C, Rouillé A, Grall A, Tréguer L, Virmaux M, et al. A cluster of pneumocystis infections among renal transplant recipients: molecular evidence of colonized patients as potential infectious sources of pneumocystis jirovecii. Clin Infect Dis. 2012;54(7): 62-71.

7. Pliquett RU, Asbe-Vollkopf A, Hauser PM, Presti LL, Hunfeld KP, Berger A, et al. A pneumocystis jirovecii pneumonia outbreak in a single kidney-transplant center: role of cytomegalovirus co-infection. Eur J Clin Microbiol Infect Dis. 2012;31(9):2429-37.

8. Yang CY, Shih CJ, Yang WC, Lin CC. Aggressive immunosuppressant reduction and long-term rejection risk in renal transplant recipients with pneumocystis jiroveci pneumonia. Exp Clin Transplant. 2012;10(4):344-9.

9. Tu GW, Ju MJ, Xu M, Rong RM, He YZ, Xue ZG, et al. Combination of caspofungin and low-dose trimethoprim/sulfamethoxazole for the treatment of severe pneumocystis jirovecii pneumonia in renal transplant recipients. Nephrology (Carlton). 2013;18(1):736-42.

10. Chen G, Zhang Z, Gu J, Qiu J, Wang C, Kung R, et al. Incidence and risk factors for pulmonary 
mycosis in kidney transplantation. Transplant Proc. 2010;42(10):4094-8.

11. Song T, Wei LP, Chen WJ, Liu P, Mai WW, Li ZZ. Imaging characteristics of pneumocystis pneumonia after renal transplantation. Ren Fail. 2010;32(1):78-84.

12. Perez-Ordoño L, Hoyo I, Sanclemente G, Ricart MJ, Cofan F, Perez-Villa F, et al. Late-onset Pneumocystis jirovecii pneumonia in solid organ transplant recipients. Transpl Infect Dis. 2014;16(2):324-8.

13. Phipps LM, Chen SCA, Kable K, Halliday CL, Firacative $\mathrm{C}$, Meyer W, et al. Nosocomial pneumocystis jirovecii pneumonia: lessons from a cluster in kidney transplant recipients. Transplantation. 2011;92(12):1327-34.

14. Hoyo I, Sanclemente G, Cervera C, Cofán F, Ricart MJ, Perez-Villa F. Opportunistic pulmonary infections in solid organ transplant recipients. Transplant Proc. 2012;44(9):2673-5.

15. Rostved AA, Sassi M, Kurtzhals JAL, Sørensen SS, Rasmussen A, Ross C, et al. Outbreak of pneumocystis pneumonia in renal and liver transplant patients caused by genotypically distinct strains of pneumocystis jirovecii. Transplantation. 2013;96(9):1-19.

16. Boer MGJ, Fijter JW, Kroon FP. Outbreaks and clustering of pneumocystis pneumonia in kidney transplant recipients: a systematic review. Med Mycol. 2011;49(7):673-80.

17. Iqbal MAH, Lim SK, Ng KP, Tan LP, Chong YB, Keng TC. Pneumocystis jirovecii pneumonia 13 years post renal transplant following a recurrent cytomegalovirus infection. Transpl Infect Dis. 2012;14(4):23-6.

18. Fritzsche C, Riebold D, Fuehrer A, Mitzner A, Klammt S, Mueller-Hilke B, et al. Pneumocystis jirovecii colonization among renal transplant recipients. Asia Pac Soc Nephrol. 2013;18(5):382-7.

19. Leth S, Jensen-Fangel S, Østergaard L, Rostved AA, Jespersen B, Søgaard OS. Pneumocystis jirovecii pneumonia in patients with end-stage renal disease: a comparison with the general population. Scand J Infect Dis. 2014;46(10):704-11.

20. De Castro N, Xu F, Porcher R, Pavie J, Molina JM, Peraldi MN. Pneumocystis jirovecii pneumonia in renal transplant recipients occurring after discontinuation of prophylaxis: a case-control study. Clin Microbiol Infect. 2010;16(9):1375-7.

21. Dizdar OS, Ersoy A, Akalin H. Pneumonia after kidney transplant: incidence, risk factors, and mortality. Exp Clin Transplant. 2014;12(3):205-11.
22. Ramalho J, Marques IDB, Aguirre AR, Pierrotti LC, Paula FJ, Nahas Wc, et al. Pneumocystis jirovecii pneumonia with an atypical granulomatous response after kidney transplantation. Transpl Infect Dis. 2014;16(2):315-9.

23. Maruschke M, Riebold D, Holtfreter MC, Sombetzki M, Mitzner S, Loebermann M, et al. Pneumocystis pneumonia (PCP) and Pneumocystis jirovecii carriage in renal transplantation patients: a single-centre experience. Wien Klin Wochenschr. 2014;126(2324):762-6.

24. Borstnar S, Lindic J, Tomazic J, Kandus A, Pikelj A, Prah J, et al. Pneumocystis jirovecii pneumonia in renal transplant recipients: a national center experience. Transplant Proc. 2013;45(4):1614-7.

25. Metan G, Bozkurt I, Koc AN. Pneumocystis jiroveci pneumonia (PCP) misdiagnosed as pandemic influenza H1N1 in a renal transplant patient. Infez Med. 2011;19(3):182-4.

26. Struijk GH, Gijsen AF, Yong SL, Zwinderman AH, Geerlings SE, Lettinga KD, et al. Risk of pneumocystis jiroveci pneumonia in patients long after renal transplantation. Nephrol Dial Transplant. 2011; 26(10):3391-8.

27. Boer MGJ, Kroon FP, le Cessie S, Fijter JW, van Dissel JT. Risk factors for Pneumocystis jirovecii pneumonia in kidney transplant recipients and appraisal of strategies for selective use of chemoprophylaxis. Transpl Infect Dis. 2011;13(6):559-69.

28. Eitner F, Hauser IA, Rettkowski O, Rath T, Lopau $\mathrm{K}$, Pliquett RU, et al. Risk factors for Pneumocystis jiroveci pneumonia $(\mathrm{PcP})$ in renal transplant recipients. Nephrol Dial Transplant. 2011;26(6):2013-7.

29. Bige N, Zafrani L, Lambert J, Peraldi MN, Snanoudj $\mathrm{R}$, Reuter $\mathrm{D}$, et al. Severe infections requiring intensive care unit admission in kidney transplant recipients: impact on graft outcome. Transpl Infect Dis. 2014;16(4):588-96.

30. Bento C, Martins LS, Almeida M, Pedroso S, Dias $\mathrm{L}$, Henriques AC, et al. A diagnosis not to forget in a long -term kidney transplant - pneumocystis jiroveci pneumonia. Port J Nephrol Hypert. 2014;28(3):260-4.

31. Chen CH, Shu KH, Ho HC, Cheng SB, Lin CC, Wei HJ, et al. A nationwide population-based study of the risk of tuberculosis in different solid organ transplantations in Taiwan. Transplant Proc. 2014;46(4):1032-5.

32. Eyüboğlu FÖ, Küpeli E, Bozbaş ŞS, Özen ZE, Akkurt ES, Aydoğan C, et al. Evaluation of pulmonary 
infections in solid organ transplant patients: 12 years of experience. Transplant Proc. 2013;45(10):3458-61.

33. Boubaker K, Gargah T, Abderrahim E, Abdallah TB, Kheder A. Mycobacterium tuberculosis infection following kidney transplantation. Biomed Res Int. 2013:1-9.

34. Kupeli E, Ulubay G, Colak T, Ozdemirel TS, Ozyurek BA, Akcay S, et al. Pulmonary complications in renal recipients after transplantation. Transplant Proc. 2011;43(2):551-3

35. Higuita LMS, Nieto-Ríos JF, Daguer-Gonzalez S, Ocampo-Kohn C, Aristizabal-Alzate A, VelezEcheverri C, et al. Tuberculose em pacientes transplantados renais: experiência de um único centro em Medellín-Colômbia, 2005-2013. J Bras Nefrol. 2014;36(4):512-8.

36. Ersan S, Celik A, Atila K, Sifil AA, Cavdar C, Soylu A, et al. Tuberculosis in renal transplant recipients. Ren Fail. 2011;33(8):753-57.

37. Nasim A, Baqi S, Zeeshan SM, Aziz T. Chronic necrotizing pulmonary aspergillosis in a renal transplant recipient. J Pak Med Assoc. 2011;61(12):1242-4.

38. Hoyo I, Linares L, Cervera C, Almela M, Marcos MA, Sanclemente G, et al. Epidemiology of pneumonia in kidney transplantation. Transplant Proc. 2010;42(8):2938-40.

39. Hoyo I, Sanclemente G, Puig de la Bellacasa J, Cofán F, Ricard MJ, Cardona M, et al. Epidemiology, clinical characteristics, and outcome of invasive aspergillosis in renal transplant patients. Transpl Infect Dis. 2014; 16(6):951-7.

40. Moloudi E, Massa E, Geogiadou E, Iosifidis E, Katsika $\mathrm{E}$, Rembelakos $\mathrm{G}$, et al. Infections related to renal transplantation requiring intensive care admission: a 20-year study. Transplant Proc. 2012;44(9):2721-3.

41. Encarnación AA, Arias LF, Cataño JC. Conferencia clínico-patológica (CPC): trasplantado renal con nódulos pulmonares. Iatreia. 2012;25(4):398-407.

42. Rizza V, Coletti G, Grimaldi A, Clemente K, Di Cocco P, D'Angelo M, et al. A rare case of herpes simplex type 1 bronchopneumonia associated with cardiomegaly in renal transplantation. Transplant Proc. 2011;43(4):1210-2.

43. Canet E, Osman D, Lambert J, Guitton C, Heng AE, Argaud L, et al. Acute respiratory failure in kidney transplant recipients: a multicenter study. Crit Care. 2011;15(2):1-10.
44. Kute VB, Patel MP, Patil SB, Shah PR, Vanikar AV, Gumber MR et al. Bronchiolitis obliterans organizing pneumonia (BOOP) after renal transplantation. Int Urol Nephrol. 2013;45(5):1517-21.

45. Jiang T, Xue F, Zheng X, Yu H, Tao XF, Xiao XS, et al. Clinical data and CT findings of pulmonary infection caused by different pathogens after kidney transplantation. Eur J Radiol. 2012;81(30):1347-52.

46. Tu G, Ju M, Zheng Y, Xu M, Rong R, Zhu D, et al. Early- and late-onset severe pneumonia after renal transplantation. Int J Clin Exp Med. 2015;8(1):132432.

47. Jabbar Z, Han TM, Gagan F. Expect the unexpected: pleuro-pulmonary melioidosis in a renal transplant recipient. Transpl Infect Dis. 2013;15(1):40-3.

48. Valdez-Ortiz R, Sifuentes-Osornio J, MoralesBuenrostro LE, Ayala-Palma H, Dehesa-López E, Alberú $\mathrm{J}$ et al. Risk factors for infections requiring hospitalization in renal transplant recipients: a cohort study. Int J Infect Dis. 2011;15(3):188-96.

49. Shih CJ, Tarng DC, Yang WC, Yang CY. Immunosuppressant dose reduction and long-term rejection risk in renal transplant recipients with severe bacterial pneumonia. Singapore Med J. 2014;55(7):3727.

50. He Q, Chen X, Lin B, Qu L, Wu J, Chen J. Late onset pulmonary lophomonas blattarum infection in renal transplantation: a report of two cases. Intern Med. 2011; 50(9):1039-43.

51. Cunha BA, Syed U, Mickail N. Renal transplant with bronchiolitis obliterans organizing pneumonia (BOOP) attributable to tacrolimus and herpes simplex virus (HSV) pneumonia. Heart Lung. 2012;41(3):310-5.

52. Gainer SM, Patel SJ, Seethamraju H, Moore LW, Knight RJ, Gaber AO. Increased mortality of solid organ transplant recipients with H1N1 infection: a single center experience. Clin Transplant. 2012;26(4):229-37.

53. Subramanian AK, Morris MI. Mycobacterium tuberculosis infections in solid organ transplantation. Am J Transplant. 2013;13 Suppl 4:68-76.

54. Zeyneloğlu P. Respiratory complications after solid-organ transplantation. Exp Clin Transplant. 2015;13(2):115-25.

55. Baas MC, Struijk GH, Moes DAR, Berk IAH, Jonkers RE, Fijter JW, et al. Interstitial pneumonitis caused by everolimus: a case-cohort study in renal transplant recipients. Transpl Int. 2014;27(5):428-36. 
56. Ou SM, Liu CJ, Teng CJ, Lin YT, Chang YS, Chiang $\mathrm{SC}$ et al. Impact of pulmonary and extrapulmonary tuberculosis infection in kidney transplantation: a nationwide population-based study in Taiwan. Transpl Infect Dis. 2012;14(50):502-9.

57. Ho TA, Rommelaere M, Coche E, Yombi JC, Kanaan N. Nontuberculous mycobacterial pulmonary infection in renal transplant recipients. Transpl Infect Dis. 2010;12(12):138-42.

58. Fraile P, Muñoz-Bellvis L, Cacharro LM, GarcíaCosmes P, Tabernero JM. Respiratory distress syndrome caused by influenza H1N1 in a patient with a simultaneous pancreas-kidney transplantation. Transplant Proc. 2013;45(9):3429-31.

59. Simkins J, Muggia V, Cohen HW, Minamoto GY. Carbapenem resistant klebsiella pneumoniae infections in kidney transplant recipients: a case-control study. Transpl Infect Dis. 2014:16(5):775-82.

60. Currie AC, Knight SR, Morris PJ. Tuberculosis in renal transplant recipients: the evidence for prophylaxis. Transplantation. 2010;90(7):695-704.

61. Meije Y, Piersimoni C, Torre-Cisneros J, Dilektasli AG, Aguado JM, ESCMID Study Group of Infection in Compromised Hosts. Mycobacterial infections in solid organ transplant recipients. Clin Microbiol Infect. 2014;20(Suppl 7):89-101.

62. Villar J, Blanco J, Campo R, Andaluz-Ojeda D, DíazDomínguez FJ, Muriel A, et al. Assessment of $\mathrm{PaO}_{2} /$ $\mathrm{FiO}_{2}$ for stratification of patients with moderate and severe acute respiratory distress syndrome. BMJ Open. 2015;5(3):1-8.

63. Fishman J. Infection in renal transplant recipients. Semin Nephrol. 2007;27(4):445-67.
64. Albuquerque JG, Lira ALBC, Lopes MVO. Fatores preditivos de diagnóstico de enfermagem em pacientes submetidos ao transplante renal. Rev Bras Enferm. 2010;63(1):98-103.

65. Filardo FA, Faresin SM, Fernandes ALG. Validade de um índice prognóstico para ocorrência de complicações pulmonares no pós-operatório de cirurgia. AMB Rev Assoc Med Bras. 2002;48(3):209-16.

66. Tomio D, da Silva RM. Pneumocistose. Arq Catarinenses Med. 2005;34(4);85-91.

67. Silva RF. Infecções fúngicas em imunocomprometidos. J Bras Pneumol. 2010;36(1):142-7.

68. Salomão R, Gonçalves AR, Caldeira M Filho, Silva E, Salomão R, Bernardo WM, et al. Diretrizes para tratamento de sepse grave/choque séptico. Rev Bras Ter Intensiva. 2011;23(2):145-57.

69. Silva DR, Gazzana MB, Dalcin PTR. Tuberculose grave com necessidade de internação em UTI. J Bras Pneumol. 2012;38(3):386-94.

\section{Endereço do primeiro autor:}

Camilla Tibiriçá da Silva Borges

Universidade Estadual de Campinas - UNICAMP

Rua Saturnino de Btrito, $323 / 2^{\circ}$ andar

Bairro: Cidade Universitária

CEP 13083-888 - Campinas - SP - Brasil

E-mail: camillatsborges@gmail.com

\section{Endereço para correspondência:}

Ana Isabela Morsch Passos

Hospital de Clínicas da UNICAMP

Rua Vital Brasil, 251

Bairro: Cidade Universitária

CEP 13083-888 - Campinas - SP - Brasil

E-mail: anaisapassos@gmail.com 